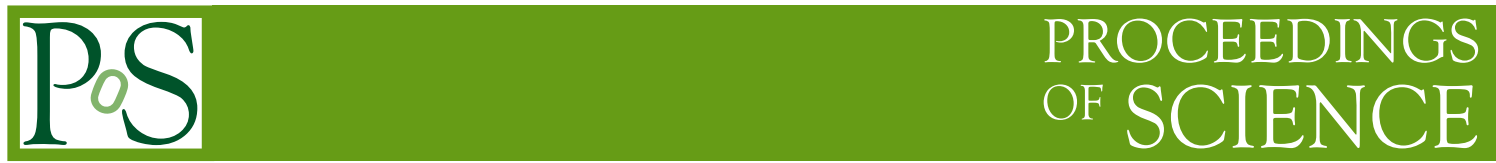

\title{
Anomalous like-sign dimuon charge asymmetry
}

\author{
Guennadi Borissov*i \\ Lancaster University, UK \\ E-mail: bgv@fnal.gov
}

I present an updated measurement of the anomalous like-sign dimuon charge asymmetry $A_{\mathrm{sl}}^{b}$ for semi-leptonic $b$-hadron decays in $9.0 \mathrm{fb}^{-1}$ of $p \bar{p}$ collisions recorded with the D0 detector at a center-of-mass energy of $\sqrt{s}=1.96 \mathrm{TeV}$ at the Fermilab Tevatron collider. The D0 collaboration obtains $A_{\mathrm{sl}}^{b}=(-0.787 \pm 0.172$ (stat) \pm 0.093 (syst) $) \%$. This result differs by 3.9 standard deviations from the prediction of the standard model and provides evidence for anomalously large $C P$ violation in semi-leptonic neutral $B$ decay. The dependence of the observed asymmetry on the muon impact parameter is consistent with the hypothesis that it originates from semi-leptonic $b$-hadron decays.

The 2011 Europhysics Conference on High Energy Physics, EPS-HEP 2011,

July 21-27, 2011

Grenoble, Rhône-Alpes, France

\footnotetext{
${ }^{*}$ Speaker.

$\dagger$ on behalf of D0 collaboration
} 
The D0 collaboration reported last year [1] the evidence of anomalous like-sign dimuon charge asymmetry $A_{\mathrm{sl}}^{b}$ using $6.1 \mathrm{fb}^{-1}$ of data:

$$
A_{\mathrm{sl}}^{b}=(-0.957 \pm 0.251 \text { (stat) } \pm 0.146 \text { (syst) }) \% .
$$

This result differs by 3.2 standard deviations from the Standard Model prediction [2]

$$
A_{\mathrm{sl}}^{b}(\mathrm{SM})=\left(-0.028_{-0.006}^{+0.005}\right) \% \text {, }
$$

In this talk I present the new result of the dimuon charge asymmetry using $9.0 \mathrm{fb}^{-1}$ of data. For this new measurement we improved the muon selection, which resulted in $13 \%$ increase of statistics for the same integrated luminosity and simultaneous $20 \%$ reduction of background from $K \rightarrow \mu$ and $\pi \rightarrow \mu$ decays. We also improved the measurement technique. In addition, we studied the dependence of the $A_{\mathrm{sl}}^{b}$ on the muon impact parameter.

The new result for $9.0 \mathrm{fb}^{-1}$ of data is:

$$
A_{\mathrm{sl}}^{b}=(-0.787 \pm 0.172 \text { (stat) } \pm 0.093 \text { (syst) }) \% \text {. }
$$

It is consistent with our previous measurement given in Eq. (1) and deviates from the Standard Model prediction by 3.9 standard deviation.

The asymmetry $A_{\mathrm{sl}}^{b}$ contains contributions from the semi-leptonic charge asymmetries $a_{\mathrm{sl}}^{d}$ and $a_{\mathrm{sl}}^{s}$ of $B^{0}$ and $B_{s}^{0}$ mesons [3], respectively:

$$
A_{\mathrm{sl}}^{b}=C_{d} a_{\mathrm{sl}}^{d}+C_{s} a_{\mathrm{sl}}^{s}, \text { with } a_{\mathrm{sl}}^{q}=\frac{\Delta \Gamma_{q}}{\Delta M_{q}} \tan \phi_{q},
$$

where $\phi_{q}$ is a $C P$-violating phase, and $\Delta M_{q}$ and $\Delta \Gamma_{q}$ are the mass and width differences between the eigenstates of the propagation matrices of the neutral $B_{q}^{0}(q=d, s)$ mesons. The coefficients $C_{d}$ and $C_{s}$ depend on the mean mixing probabilities and the production fractions of $B^{0}$ and $B_{s}^{0}$ mesons. We use the production fractions measured at LEP as averaged by the Heavy Flavor Averaging Group (HFAG) [4] and obtain

$$
C_{d}=0.594 \pm 0.022, C_{s}=0.406 \pm 0.022 .
$$

The constraint imposed by the new value of $A_{\mathrm{sl}}^{b}$ is presented in Fig. 1. The constraints from other existing measurements of $a_{\mathrm{sl}}^{d}[4]$ and $a_{\mathrm{sl}}^{s}$ [5] are also shown.

The asymmetry $A_{\mathrm{sl}}^{b}$ is produced by muons from direct semi-leptonic decays of $b$ quarks. A distinctive feature of these muons is the large impact parameter (IP) of their trajectories with respect to the primary vertex. This feature can be used to verify the origin of observed asymmetry [6]. The dominant source of background muons comes from decays to muon of long-lived charged hadrons, $\pi \rightarrow \mu$ and $K \rightarrow \mu$. These muons are denoted below as " $L$ " muons. The majority of these hadrons is produced in the primary interactions, and the tracks of $L$ muons have small impact parameters if the original hadron decays outside the tracking volume.

Figure 2 shows the muon IP distribution in data and in simulation. The shaded histogram shows the contribution from $L$ muons in simulation, which decreases significantly for increasing values of the muon IP. The background can therefore be significantly suppressed by selecting muons with large impact parameter. 


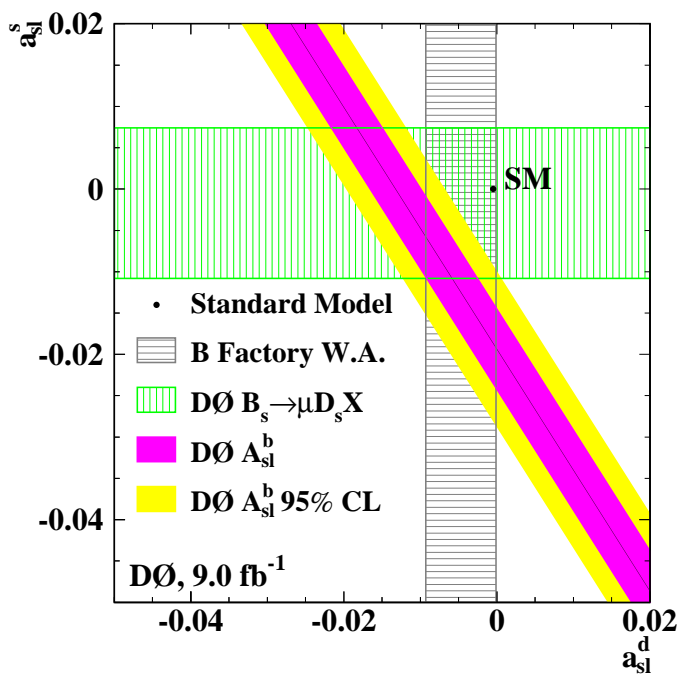

Figure 1: Comparison of $A_{\mathrm{sl}}^{b}$ in data with the SM prediction for $a_{\mathrm{sl}}^{d}$ and $a_{\mathrm{sl}}^{s}$. Also shown are the measurements of $a_{\mathrm{sl}}^{d}$ [4] and $a_{\mathrm{sl}}^{s}$ [5]. The error bands represent the \pm 1 standard deviation uncertainties on each individual measurement. The 95\% C.L. band is also given for this $A_{\mathrm{sl}}^{b}$ measurement.

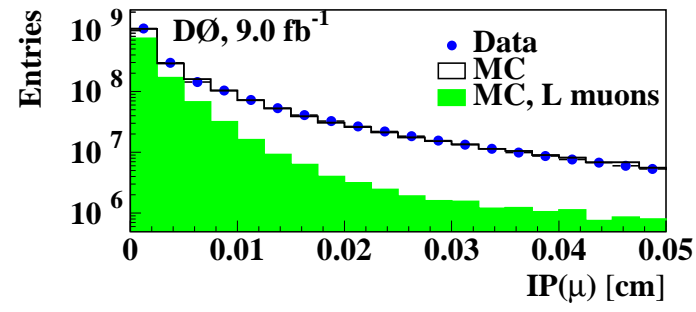

Figure 2: The muon IP distribution in the inclusive muon sample (bullets). The solid line represents the muon IP distribution in simulation. The shaded histogram is the contribution from $L$ muons in simulation.

To test the origin of the like-sign dimuon charge asymmetry we perform two complimentary measurements requiring the muon impact parameter to be larger or smaller than $120 \mu \mathrm{m}$. The contributions of $a_{\mathrm{sl}}^{d}$ and $a_{\mathrm{sl}}^{s}$ in the $A_{\mathrm{sl}}^{b}$ asymmetry change significantly when we select muons with IP above or below given threshold:

$$
\begin{aligned}
& A_{\mathrm{sl}}^{b}=(0.728 \pm 0.018) a_{\mathrm{sl}}^{d}+(0.272 \pm 0.018) a_{\mathrm{s} 1}^{s} \text { for } \mathrm{IP}>120 \mu \mathrm{m} \\
& A_{\mathrm{sl}}^{b}=(0.397 \pm 0.022) a_{\mathrm{sl}}^{d}+(0.603 \pm 0.022) a_{\mathrm{s} 1}^{s} \text { for } \mathrm{IP}<120 \mu \mathrm{m}
\end{aligned}
$$

The change of these contributions is influenced by the significant difference in the oscillation frequency of $B^{0}$ and $B_{s}^{0}$ mesons.

We obtain

$$
\begin{aligned}
& A_{\mathrm{sl}}^{b}=(-0.579 \pm 0.210(\text { stat }) \pm 0.094(\text { syst })) \% \text { for } \mathrm{IP}>120 \mu \mathrm{m} \\
& A_{\mathrm{sl}}^{b}=(-1.14 \pm 0.37 \text { (stat) } \pm 0.32(\text { syst })) \% \text { for } \mathrm{IP}<120 \mu \mathrm{m}
\end{aligned}
$$




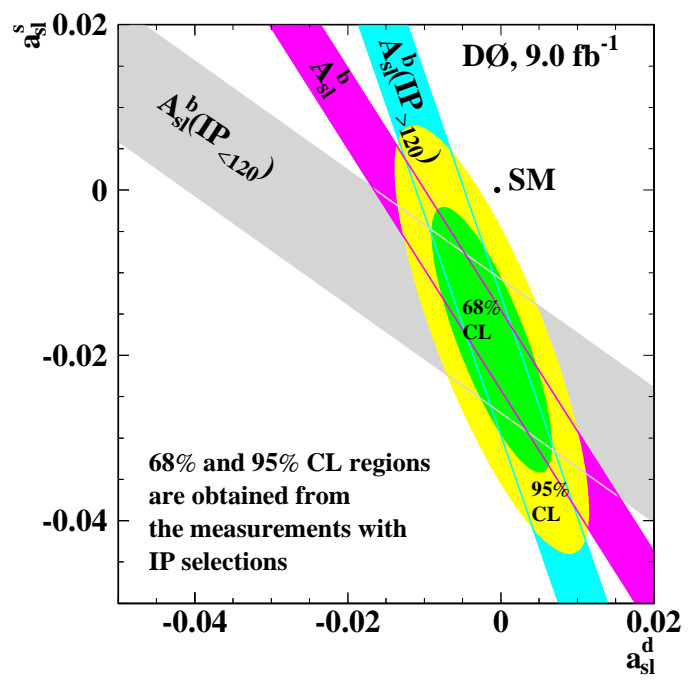

Figure 3: Measurements of $A_{\mathrm{sl}}^{b}$ with different muon IP selections in the $\left(a_{\mathrm{sl}}^{d}, a_{\mathrm{sl}}^{s}\right)$ plane. The bands represent the \pm 1 standard deviation uncertainties on each individual measurement. The ellipses represent the $68 \%$ and 95\% two-dimensional C.L. regions, respectively, of $a_{\mathrm{sl}}^{s}$ and $a_{\mathrm{sl}}^{s}$ values obtained from the measurements with IP selections.

From these results we obtain the separate values of $a_{\mathrm{sl}}^{d}$ and $a_{\mathrm{sl}}^{s}$ :

$$
a_{\mathrm{sl}}^{d}=(-0.12 \pm 0.52) \%, a_{\mathrm{sl}}^{s}=(-1.81 \pm 1.06) \%,
$$

which are consistent with the world average values of these quantities [4]. Figure 3 presents the results of the IP study in the $\left(a_{\mathrm{sl}}^{d}, a_{\mathrm{sl}}^{s}\right)$ plane together with the result (3) of the $A_{\mathrm{sl}}^{b}$ measurement using all like-sign dimuon events. The ellipses represent the $68 \%$ and $95 \%$ two-dimensional C.L. regions, respectively, of $a_{\mathrm{sl}}^{s}$ and $a_{\mathrm{sl}}^{s}$ values obtained from the measurements with IP selections.

In conclusion, the new measurement of the like-sign dimuon charge asymmetry $A_{\mathrm{sl}}^{b}$ is performed by the D0 experiment using $9 \mathrm{fb}^{-1}$ of data. The obtained result deviates from the SM prediction by 3.9 standard deviations. The dependence of $A_{\mathrm{sl}}^{b}$ on muon impact parameter is consistent with the hypothesis that the dimuon charge asymmetry arises from semileptonic $b$-hadron decays.

\section{References}

[1] V.M. Abazov et al. (D0 Collaboration), Phys. Rev. D 82, 032001 (2010); V.M. Abazov et al. (D0 Collaboration), Phys. Rev. Lett. 105, 081801 (2010).

[2] A. Lenz and U. Nierste, J. High Energy Phys. 0706, 072 (2007).

[3] Y. Grossman et al., Phys. Rev. Lett. 97, 151801 (2006).

[4] D. Asner et al., Heavy Flavor Averaging Group (HFAG), arXiv:1010.1589 [hep-ex] (2010).

[5] V.M. Abazov et al. (D0 Collaboration), Phys. Rev. D 82, 012003 (2010).

[6] M. Gronau, J. L. Rosner, Phys. Rev. D 82, 077301 (2010). 